\title{
DIE LAGEREFLEXE DES MENSCHEN
}

KLINISCHE UNTERSUCHUNGEN ÜBER HALTUNGS- UND

STELLREFLEXE UND VERWANDTE PHÄNOMENE

VON

\section{HANS HOFF}

DR. MED. SEKUNDARARZT DER PSYCHIATRISCH-NEUROLOGISCHEN KLINIK DER UNIVERSITÄT WIEN

\section{PAUL SCHILDER}

UND DR. MED. ET. PHIL., PROF., ASSISTENT DER PSYCHIATRISCH-NEUROLOGISCHEN KLINIK DER UNIVERSITÄT WIEN

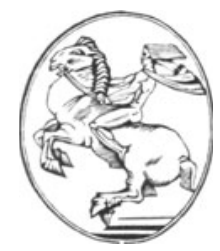


ISBN 978-3-7091-2149-8 ISBN 978-3-7091-2193-1 (eBook)

DOI 10.1007/978-3-7091-2193-1

ALLE RECHTE, INSBESONDERE DAS DER ÜBERSETZUNG

IN FREMDE SPRACHEN, VORBEHALTEN

Copyright 1928 by Springer-Verlag Wien

Ursprünglich erschienen bei Julius Springer, Vienna 1928. 


\section{Vorwort}

Die bahnbrechenden experimentellen Untersuchungen von MAGNUS und DE KLEYN haben neue Erkenntnisse über das Zentralnervensystem gebracht. Der Versuch lag nahe, diese Erkenntnisse der Klinik dienstbar zu machen. Nach vereinzelten Erfahrungen anderer hat Simons den ersten systematischen Vorstoß gewagt, aber erst durch die Untersuchungen von Goldstein, dem Fischer und Wodak, Zingerle und wir selbst folgten, wurde klar, welch bedeutsames Neuland sich hier für die klinische Neurologie erschließe. Darüber hinaus erwuchsen besonders durch GoLdsTeIns Arbeit theoretische Erkenntnisse allgemeineren Charakters, welche, das Empfinden und Wahrnehmen betreffend, sich im Versuche am Tiere der Feststellung entzogen hatten. Gewiß, hier liegt noch nichts Abgeschlossenes und Endgültiges vor. Aber das Tatsachenmaterial ist bereits ein reiches und ist nicht leicht übersehbar. Manches scheint der praktischen Verwertung am Krankenbett entgegenzureifen. So versuchen wir auf Grund mehrjähriger Beschäftigung mit dem Gegenstand an dem reichen Material der Wiener Universitätsklinik, das bisher Geförderte zusammenzufassen, wohl wissend, daß die klinische Methodik dem Standardwerk von MAGNus noch nichts Gleichwertiges zur Seite setzen kann. Wir haben uns um die Darstellung der Probleme besonders bemüht und hoffen, so einer künftigen Forschung den Weg zu bereiten. Von unserem kasuistischen Material haben wir nur einen kleinen Bruchteil mitgeteilt; wir könnten die Zahl der Krankengeschichten leicht vervielfachen: Handelt es sich doch keineswegs um Phänomene mit Seltenheitswert. Wir suchen das Typische und klinisch Verwertbare. Unseren Absichten entsprechend, geben wir nur einen kurzen Abriß der Anatomie und der experimentellen Erfahrungen und verweisen denjenigen, der sich in den Gegenstand vertiefen will, eindringlich auf das Buch von Magnus. Unsere eigenen Erfahrungen an Kindern sind spärlich. Auch dieser Abschnitt ist vorwiegend referierend. Hingegen stützen wir uns in den übrigen Teilen des Buches auf eigene Erfahrungen.

Wien, im Oktober 1927

Die Verfasser 


\section{Inhaltsverzeichnis}

I. Haltungs- und Stellreflexe beim Tier ............... Seite Anhang: Einige anatomische Bemerkungen über das Nucleus-

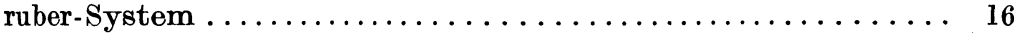

II. Haltungs- und Stellreflexe beim Menschen ......... 22

a) Haltungs- und Stellreflexe beim Fötus und beim Kinde.... 22

b) Haltungs- und Stellreflexe beim Erwachsenen .......... 38

III. Zur Technik der Darstellung der Haltungs- und Stellreflexe beim Gesunden und Kranken .............6.67

IV. Klinik der Haltungs- und Stellreflexe ............ 69

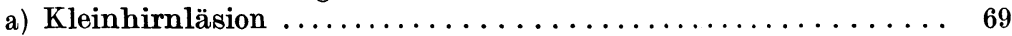

b) Tonusverlust $\ldots \ldots \ldots \ldots \ldots \ldots \ldots \ldots \ldots \ldots \ldots \ldots \ldots \ldots \ldots \ldots$

c) Chorea ................................. 109

d) Delirium tremens ............................ 111

e) Tabes dorsalis .............................. 114

f) Athetose und striäre Hyperkinesen . . . . . . . . . . 120

g) Hemiplegie ............................ 122

h) Bemerkungen über Gangbewegungen und Zusatzreize ..... 127

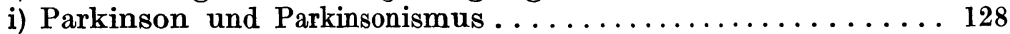

j) Die Hirnrindenbeziehungen der Haltungs- und Stellreflexe... 138

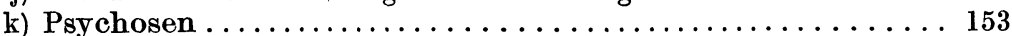

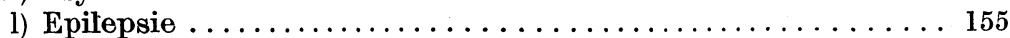

V. Tabelle der wichtigsten in diesem Buche verwerteten

Phänomene ............................. 156

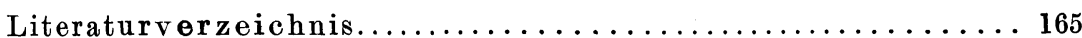

